\title{
Urologen begrüßen informierte Patienten
}

An Schlagzeilen mangelte es nicht: Eine jüngst von der Bertelsmann Stiftung und der Barmer GEK veröffentlichte Studie, wonach Ärzte informierte Patienten kritisch sehen, fand regen Widerhall in den Medien. Der BDU und die DGU betonen angesichts der Online-Umfrage unter rund 800 niedergelassenen Ärzten unterschiedlicher Fachbereiche in Deutschland, dass sie den informierten Patienten ausdrücklich begrüßen und weisen aus diesem Grund auf das eigene umfängliche Engagement bei der medizinischen Aufklärung der Bevölkerung und die Vielfalt ihrer eigenen Informationsangebote hin. Erst vor Kurzem wurde das Aufklärungspaket der Urologen durch die innovative „Online Entscheidungshilfe Prostatakrebs" noch einmal erweitert.

„Für uns steht das legitime Informationsbedürfnis der Patienten nicht infrage“, sagt BDU-Präsident Dr. Axel Schroeder. „Aber natürlich wissen wir, und darauf weist auch die Umfrage der Bertelsmann Stiftung und der Barmer GEK hin, dass es für den medizinischen Laien, vor allem bei der selbstständigen Internetrecherche schwierig ist, seriöse Quellen zu identifizieren. Medizinisch geprüfte Informationen sind aber gerade für onkologische Patienten, allen voran Prostatakrebspatienten, die wir in der Urologie in einer großen Zahl betreuen, besonders wichtig.“

Gerade deshalb engagieren sich der BDU und die DGU seit Jahren bei der Erstellung eigener Informationsangebote. Dazu zählen das Engagement bei den Patientenleitlinien zum Prostata-, Nieren- und Harnblasenkrebs, das umfangreiche Angebot an eigenen Patientenbroschüren zu relevanten urologischen Erkrankungen und deren Prävention, das Internetportal der deutschen Urologen (www.urologenportal.de) und die speziell für die männlichen Adoleszenten konzipierte Webseite www. jungensprechstunde.de ebenso wie die Internetkampagne zum Hodenhochstand, mit der 2015 gezielt junge Eltern angesprochen wurden. Absolut neue Wege der individuellen Beratung geht die interaktive „Online Entscheidungshilfe Prostatakrebs" der PatientenAkademie der Deutschen Urologen (www.entscheidungshil- 
fe-prostatakrebs.info), die Betroffene und ihre betreuenden Urologen bei der Wahl der individuell richtigen Therapie unterstützt. „Die Tatsache, dass wir als Fachgesellschaft und Berufsverband eine eigene PatientenAkademie unterhalten, zeigt, welchen hohen Stellenwert Deutschlands Urologen der wissenschaftlich begleiteten Patientenaufklärung beimessen“, sagt der DGU-Pressesprecher und 2. Vorsitzende der PatientenAkademie, Prof. Christian Wülfing.

Der BDU verweist zudem auf seinen Selektivvertrag zur Aktive Surveillance, der einen aufklärungsintensiven Behandlungspfad beim Prostatakarzinom unterstützt, sowie auf seinen speziellen PCaAufklärungsleitfaden, der das Arzt-Patienten-Gespräch auf hohem Niveau standardisiert. BDU-Präsident Dr. Schroeder: „Nicht zuletzt sei an dieser Stelle die jahrelange enge Zusammenarbeit der urologischen Verbände mit der Selbsthilfe, gerade mit dem Bundesverband Prostatakrebs Selbsthilfe (BPS), genannt, die ebenfalls dazu beiträgt, dass Männer sowohl bei der Früherkennung des Prostatakarzinoms (PCa) als auch bei der Behandlung einer Prostatakrebserkrankung eine informierte Entscheidung treffen können.“

„Mit diesen vertrauenswürdigen Aufklärungsangeboten unterstützen DGU und BDU das Informationsbedürfnis der urologischen Patienten und Patientinnen jeden Alters und beiderlei Geschlechts umfänglich. Befürchtungen von Ärzten, Selbstinformation könne beim Patienten übersteigerte Erwartungen wecken und das Vertrauen zum Arzt gefährden, wie sie von der aktuellen Umfrage der Bertelsmann Stiftung und der Barmer GEK fachübergreifend erhoben wurden, wirken wir damit entgegen. Denn ein seriös informierter Patient kann mit seinem Arzt vertrauensvoll und zielorientiert über seine Erkrankung und Therapieoptionen diskutieren“, so DGU-Pressesprecher Prof. Wülfing.

Fortgesetzt wird das Informationsprogramm der Urologen mit dem traditionellen Patientenforum am 1. Oktober 2016 auf dem 68. DGU-Kongress in Leipzig, zu dem DGU-Präsident Prof. Kurt Miller bereits jetzt alle Interessierten herzlich ins Congress Center Leipzig einlädt.

Nach einer Pressemitteilung ( $D G U / B D U)$ 